\title{
Polarisation in A-type stars with circumstellar shells
}

\author{
H.C. Bhatt \\ Indian Institute of Astrophysics, Bangalore 560034, India \\ e-mail: hcbhatt@iiap.ernet.in \\ Received March 13; accepted May 17, 1996
}

\begin{abstract}
We present the results of optical linear polarisation measurements of about 30 A-type stars that are known to be either Ae/A shell stars or have shown anomalous emission in the infrared. Compared with the general population of A-type stars, stars of this group are found to have polarisation that is also anomalous and is suggested to be circumstellar in origin.
\end{abstract}

Key words: polarisation — stars: emission-line — stars: circumstellar matter — infrared: stars

\section{Introduction}

A large number of A-type stars observed by the Infrared Astronomical Satellite (IRAS) have been found to have infrared excesses (e.g. Aumann 1985; Coté 1987). The excess infrared radiation is emitted by matter (gas and dust) in the circumstellar regions around these stars. The star $\beta \mathrm{Pic}$ is one such anomalous infrared emitter. This star also exhibits a "shell" spectrum, characterized by the simultaneous presence of both broad and sharp lines in the spectrum. The broad lines are stellar in origin, while the sharp lines are understood to be produced in a circumstellar shell. From a study of a sample of Ae/A shell stars Jaschek et al. (1986) showed that in stars of this group the proportion of anomalous infrared emitters (AIE) is very high, suggesting a close link between "IRAS excesses" and "shell-type characteristics". Jaschek et al. (1991) have examined all A-type stars contained in the Bright Star Catalogue, Fourth Edition, for IRAS infrared excesses; and a sample of them in detail for shell characteristics. This study confirms their earlier findings about the correlation between anomalous infrared emission and shell characteristics. Jaschek et al. (1991) also give lists of A-type AIEs and Ae/A-type shell stars.

The shell characteristics in the spectrum are attributed to circumstellar gas shells within a few stellar radii, while the IRAS infrared excesses are due to cold (50-400 K) dust much further away from the star. The observed correlation between anomalous infrared emission and shell characteristics therefore implies that A-type stars having circumstellar matter of one kind are also likely to have the other, and Ae/A shell stars are physically related to A-type AIEs (Jaschek et al. 1986). A majority, if not all, of A-type AIEs could be expected to show "shell characteris- tics" upon spectroscopic measurements at high dispersion, not yet available for most of them.

Circumstellar shells can also cause another observational effect. Light from the central star can get polarised upon scattering by matter in the circumstellar shells. For example, the polarisation observed in the light of young $\mathrm{T}$ Tauri stars and the Herbig $\mathrm{Ae} / \mathrm{Be}$ stars that are generally associated with dusty cloud material, is ascribed to the presence of circumstellar matter in these systems (e.g. Bastien 1988). A-type AIEs and A-type shell satrs also have circumstellar matter surrounding them, so it may be useful to look for polarisation in these stars as another signature of its presence. In this paper, we present the results of our polarisation measurements for about $30 \mathrm{~A}$ type AIEs and Ae/A-type shell stars from the lists of such stars given by Jaschek et al. (1991). These are then compared with measurements for normal A-type stars in the Bright Star Catalogue. We find that the A-type AIEs and shell stars exhibit anomalous polarisation.

\section{Observations}

The linear polarisation measurements in the optical $V$ band were made with a fast star-and-sky chopping polarimeter coupled at the $f / 13$ cassegrain focus of the 102 $\mathrm{cm}$ telescope at the Vainu Bappu Observatory, Kavalur, on several nights during 1992-93. The instrument and the data reduction method have been described elsewhere (Jain \& Srinivasulu 1991). A dry ice cooled EMI 9658$\mathrm{R}$ (extended S-20) photomultiplier tube and the Fernie (1974) combination of glass filters were used for the observations. Unpolarised standard stars (Serkowski 1974) were measured to determine the instrumental polarisation. It was found to be $\sim 0.1 \%$, and was subtracted vectorially 
Table 1. Polarisation measurements of A-type stars

\begin{tabular}{|c|c|c|c|c|c|c|c|c|c|c|c|}
\hline $\begin{array}{l}\mathrm{HR} \\
\text { no. }\end{array}$ & $\begin{array}{c}\text { Date of } \\
\text { observation }\end{array}$ & Sp.Type & $V$ & $B-V$ & $E(B-V)$ & $m-M$ & $\begin{array}{l}P \\
\%\end{array}$ & $\begin{array}{l}\epsilon_{\mathrm{p}} \\
\%\end{array}$ & $\begin{array}{l}\theta \\
\circ\end{array}$ & $\begin{array}{l}\epsilon_{\theta} \\
\circ\end{array}$ & Remarks \\
\hline 10 & 29 Oct 92 & $\mathrm{~A} 6 \mathrm{Vn}$ & 6.19 & 0.14 & -0.03 & 4.0 & 0.19 & 0.07 & 160 & 11 & $\mathrm{Sh}$ \\
\hline 451 & 29 Oct 92 & A3V & 5.63 & 0.07 & -0.01 & 3.8 & 0.07 & 0.06 & 20 & 24 & AIE \\
\hline 622 & 29 Oct 92 & A5III & 3.00 & 0.14 & -0.01 & 1.9 & 0.30 & 0.10 & 34 & 9 & AIE \\
\hline 664 & 29 Oct 92 & A1Vnn & 4.01 & 0.02 & 0.01 & 2.7 & 0.07 & 0.06 & 106 & 25 & AIE \\
\hline 2148 & 27 Jan 93 & $\mathrm{~A} 2 \mathrm{e}$ & 4.93 & 0.24 & 0.19 & 2.8 & 0.22 & 0.08 & 19 & 10 & $\mathrm{Sh}, \mathrm{AIE}$ \\
\hline 2174 & 27 Jan 93 & A3Vn & 5.73 & 0.07 & 0.01 & 3.9 & 0.09 & 0.06 & 103 & 19 & $\mathrm{Sh}, \mathrm{AIE}$ \\
\hline 2996 & 27 Mar 92 & A2Iabe & 3.96 & 0.18 & 0.20 & 10.1 & 1.51 & 0.12 & 94 & 2 & AIE \\
\hline 3479 & 27 Jan 93 & B2III & 5.76 & -0.15 & 0.09 & 9.1 & 0.34 & 0.10 & 84 & 9 & $\mathrm{Sh}$ \\
\hline \multirow[t]{2}{*}{3589} & 01 Jan 93 & A8Vn & 6.07 & & & 3.6 & 0.39 & 0.09 & 137 & 6 & $\mathrm{Sh}$ \\
\hline & 27 Jan 93 & & & & & & 0.17 & 0.05 & 137 & 9 & \\
\hline 3744 & 27 Jan 93 & $\mathrm{~A} 2 \mathrm{~V}$ & 6.54 & 0.05 & 0.00 & 4.9 & 0.11 & 0.08 & 129 & 22 & $\mathrm{Sh}$ \\
\hline 3921 & 31 Mar 92 & A2 & 6.24 & 0.04 & 0.00 & 0.0 & 0.17 & 0.10 & 46 & 17 & $\mathrm{Sh}$ \\
\hline 3989 & 31 Mar 92 & $\mathrm{~A} 1 \mathrm{~V}$ & 5.91 & 0.02 & 0.01 & 4.6 & 0.06 & 0.07 & & & $\mathrm{Sh}$ \\
\hline 4110 & 31 Mar 92 & A5Ia & 4.66 & 0.51 & 0.46 & 11.0 & 2.06 & 0.30 & 146 & 4 & AIE \\
\hline 4295 & 31 Mar 92 & A1V & 2.37 & -0.02 & -0.03 & 1.1 & 0.27 & 0.10 & 91 & 11 & AIE \\
\hline 4368 & 30 Mar 92 & A7IVn & 4.47 & 0.21 & 0.03 & 2.6 & 0.43 & 0.09 & 49 & 6 & $\mathrm{Sh}$ \\
\hline 4398 & 30 Mar 92 & A4:p & 5.79 & 0.01 & -0.10 & 3.8 & 0.92 & 0.32 & 28 & 10 & $\mathrm{Sh}$ \\
\hline 4534 & 27 Mar 92 & A3V & 2.14 & 0.09 & 0.01 & 0.3 & 0.46 & 0.11 & 82 & 7 & AIE \\
\hline 4554 & 31 Mar 92 & $\mathrm{~A} 0 \mathrm{Ve}$ & 2.44 & 0.00 & 0.02 & 1.4 & 0.10 & 0.11 & & & $\mathrm{Sh}$ \\
\hline 4733 & 31 Mar 92 & F0p & 4.95 & 0.27 & -0.02 & 2.9 & 0.13 & 0.07 & 78 & 15 & $\mathrm{Sh}$ \\
\hline 5112 & 30 Mar 92 & A5V & 4.70 & 0.12 & -0.03 & 2.6 & 0.02 & 0.11 & & & $\mathrm{Sh}$ \\
\hline 5175 & 27 Mar 92 & $\mathrm{~A} 3 \mathrm{~m}$ & 5.91 & 0.29 & 0.15 & 3.4 & 0.28 & 0.08 & 40 & 8 & AIE \\
\hline 5435 & 28 Mar 92 & A7III & 3.03 & 0.19 & 0.01 & 1.8 & 0.16 & 0.05 & 32 & 10 & AIE \\
\hline 5774 & 30 Mar 92 & A5V & 5.02 & 0.07 & 0.08 & 2.7 & 0.04 & 0.10 & & & $\mathrm{Sh}$ \\
\hline 5793 & 27 Jan 93 & AOV & 2.23 & -0.02 & 0.00 & 1.2 & 0.27 & 0.23 & 45 & 25 & AIE \\
\hline 5798 & 28 Mar 92 & A0V & 5.44 & 0.00 & 0.02 & 4.4 & 0.10 & 0.12 & & & AIE \\
\hline 5999 & 27 Feb 93 & A7IVe & 7.05 & 0.36 & 0.18 & 4.7 & 0.58 & 0.11 & 168 & 5 & $\mathrm{Sh}, \mathrm{AIE}$ \\
\hline 6123 & 30 Mar 92 & A5V & 5.52 & & & 3.4 & 0.32 & 0.14 & 2 & 12 & $\mathrm{Sh}$ \\
\hline 6232 & 27 Mar 92 & A3V & 6.10 & 0.14 & 0.06 & 4.1 & 0.53 & 0.08 & 96 & 4 & $\mathrm{Sh}, \mathrm{AIE}$ \\
\hline 6273 & 27 Mar 92 & A7IIIm: & 6.35 & 0.21 & 0.03 & 4.5 & 0.50 & 0.12 & 168 & 7 & AIE \\
\hline 6507 & 31 Mar 92 & A8V & 5.44 & 0.22 & 0.01 & 2.9 & 0.24 & 0.06 & 136 & 7 & $\mathrm{Sh}$ \\
\hline 6629 & 28 Mar 92 & A0V & 3.75 & 0.04 & 0.06 & 2.6 & 0.17 & 0.06 & 32 & 9 & AIE \\
\hline 6631 & 31 Mar 92 & A2Ib & 4.81 & 0.26 & 0.28 & 9.0 & 1.92 & 0.08 & 5 & 1 & AIE \\
\hline 6723 & 30 Apr 92 & A2Vn & 4.45 & 0.02 & -0.03 & 2.9 & 0.46 & 0.29 & 149 & 18 & $\mathrm{Sh}$ \\
\hline 6864 & 28 Mar 92 & A2III & 6.16 & 0.26 & 0.23 & 4.8 & 0.79 & 0.16 & 137 & 6 & $\mathrm{Sh}, \mathrm{AIE}$ \\
\hline
\end{tabular}

from the polarisation measurements for the programme stars. The zero of the position angle was determined by observing polarised standard stars (Hsu \& Breger 1982) each night.

\section{Results}

The results are listed in Table 1 . In Table 1, the HR number of the star is given in Col. 1, the date of observation in Col. 2, spectral type in Col. 3, $V$ magnitude in Col. 4, the colour $B-V$ in Col. 5, colour excess $E(B-V)$ in Col. 6,the distance modulus $m-M$, in magnitudes, in Col. 7. The observed degree of polarisation $P(\%)$ and the position angle $\theta\left(^{\circ}\right)$ are given in Cols. 8 and 10, while the probable errors in the measurements of polarisation $\epsilon_{\mathrm{p}}$ and position angle $\epsilon_{\theta}$ are given in Cols. 9 and 11 respectively. The distance modulus has been corrected for extinction by using a value of 3.0 for the ratio of total to selective extinction $A_{V} / E(B-V)$. The extinction values are generally quite small for the programme stars. Negative values of $E(B-V)$ in Col. 6 have been called zero to derive the corrected magnitudes of the stars. For stars HR 3589 and $6123, B-V$ values are not available. They have been assigned $E(B-V)=0.0$ while estimating their distance modulii, which turn out to be small $\sim 3.5$. Intrinsic colours and absolute magnitudes for stars were taken from Schmidt-Kaler (1965). Column 12 indicates whether the star is a known anomalous infrared emitter (AIE) and/or a shell (Sh) star.

\section{Discussion}

The degree of polarisation measured for our programme stars varies from a negligibly low value of $0.02 \%$ (for HR 5112 ) to values as large as $2.06 \%$ (for HR 4110). The largest values are generally observed for distant stars with distance modulii $m-M>5$. All the stars observed are bright stars from the Bright Star Catalogue, and most of them are nearby objects within $100 \mathrm{pc}$ (distance modulus $<5$ ). At such short distances the contribution of the interstellar polarisation to the observed polarisation is expected to be small $\sim 0.1 \%$. The interstellar contribution increases with distance and should be subtracted from the observed polarisation. This is, however, not possible for the programme stars individually, as the interstellar polarisation in the direction of the programme stars, as a 
function of distance, is generally not known. We therefore make a statistical comparison between the polarisation observed for the programme stars and other normal A-type stars in the Bright Star Catalogue with a similar magnitude range. For comparison, polarisation measurements

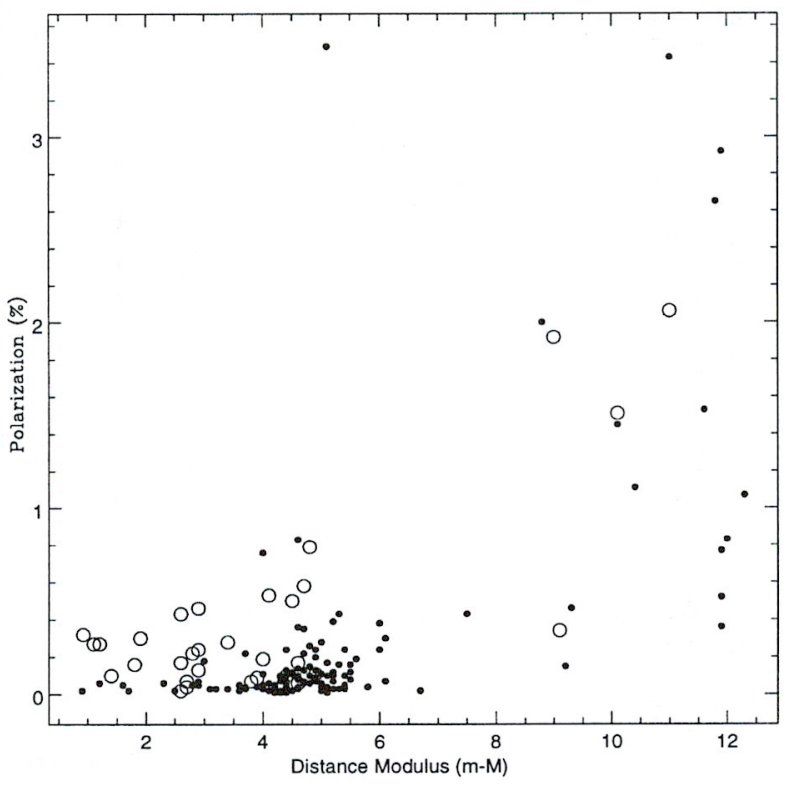

Fig. 1. Plot of degree of polarisation against the distance modulus for the A-type shell stars and anomalous infrared emitters (O) and the general population of A-type stars ( )

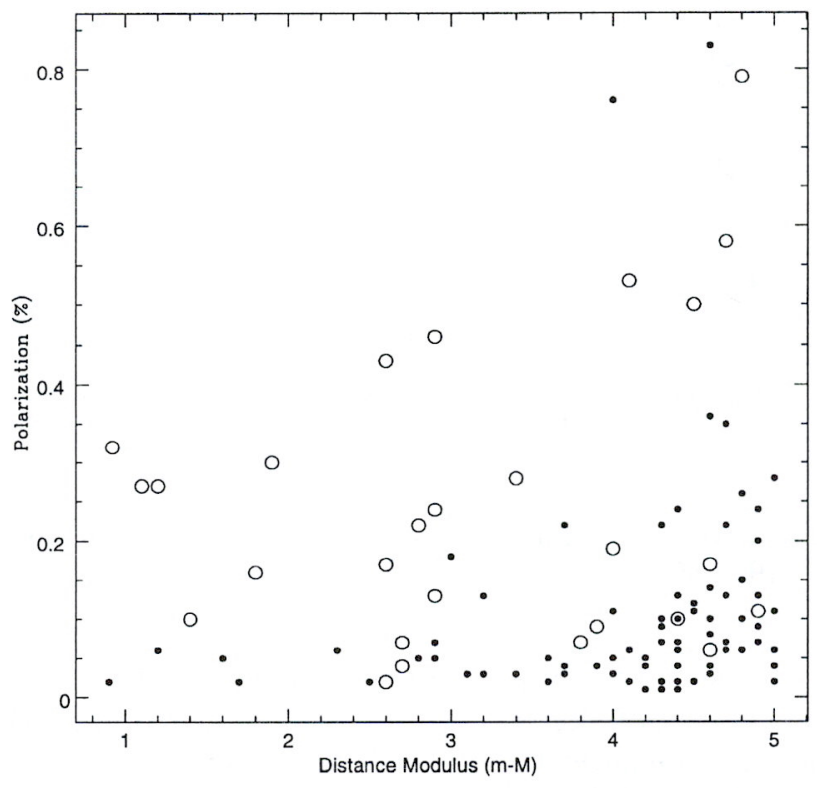

Fig. 2. Same as Fig. 1, for stars with distance modulii $m-M$ $\leq 5.0$

for normal A-type stars have been taken from Mathewson \& Ford (1970). For 130 A-type stars in the Bright
Star Catalogue that are neither AIEs nor shell stars, polarisation measurements are listed in Mathewson \& Ford (1970). These stars also show a range of values of the degree of polarisation which generally increases with distance as is expected of interstellar polarisation. In Fig. 1 we plot the observed degree of polarisation against the distance modulus for our programme stars (A-type AIEs and Ae/A-type shell stars) as well as the general population of normal A-type stars from Mathewson \& Ford (1970). Now the difference between the polarimetric behaviour of the two samples of stars is clear. For distance modulii < 5.0 , our programme stars have polarisation values generally larger than the normal A-type stars at comparable distances. Only one star belonging to the general population of normal A-type stars, HR 6081 with a distance modulus of 5.1, shows exceptionally high value (3.49\%) of polarisation for its distance. But this star also suffers an exceptionally large reddening $(E(B-V)=0.72)$ due to the nearby interstellar cloud L 1680 in Scorpius. For larger distances $(m-M \gg 5.0)$ the interstellar component begins to dominate the observed polarisation and the programme stars show polarisation values similar to those of the general population of A-type stars. The distinction between the A-type AIE and Ae/A-type shell stars and the normal A-type stars is more clearly seen in Fig. 2 where only stars with distance modulii $\leq 5.0$ are plotted. A two-sided Kolmogorov-Smirnoff test shows that the two samples are different to $99.78 \%$. The A-type AIEs and shell stars show systematically higher values of polarisation than the normal stars. For clarity in Figs. 1 and 2, the error bars have not been shown explicitly. For the degree of polarisation, these are listed in Table 1 for the programme stars, and noted to be $\sim 0.03 \%$ in Mathewson \& Ford (1970), for the normal bright A-type stars. Any errors in the estimates of the distance modulii will not cause a systematic separation on the polarisation axes in Figs. 1 and 2, which is observed to be much larger than the errors in the polarisation measurements. Therefore, the observed high values of polarisation for these nearby (distance $<100 \mathrm{pc}$ ) stars can not be accounted for by normal interstellar polarisation and must be circumstellar in origin. Circumstellar matter around the A-type shell stars and the AIEs can cause polarisation by the process of scattering the light from the central star. The wavelength dependence of polarisation under such situations may in general be different from the wavelength dependence of interstellar polarisation that is found to follow the empirical law given by Serkowski (1973) and shows a peak at $\lambda \sim 0.54 \mu$ in the $V$ band. The interstellar polarisation position angles are generally independent of wavelength. We have multi-wavelength polarisation measurements for two of our programme stars, HR 5999 and HR 6864 that show relatively large degree of polarisation. For these stars, polarisation was measured in the $B(0.44$ $\mu), V(0.55 \mu), R(0.70 \mu)$ and $I(0.90 \mu)$ bands. The 
results are listed in Table 2 . The wavelength dependence

Table 2. Multi-wavelength measurements for HR 5999 and HR 6864

\begin{tabular}{lllllllll}
\hline Wave-band & \multicolumn{4}{c}{ HR 5999 } & \multicolumn{4}{c}{ HR 6864 } \\
& $P$ & $\epsilon_{\mathrm{p}}$ & $\theta$ & $\epsilon_{\theta}$ & $P$ & $\epsilon_{\mathrm{p}}$ & $\theta$ & $\epsilon_{\theta}$ \\
& $\%$ & $\%$ & ${ }^{\circ}$ & ${ }^{\circ}$ & $\%$ & $\%$ & ${ }^{\circ}$ & ${ }^{\circ}$ \\
\hline$B$ & 0.77 & 0.20 & 182 & 8 & 0.77 & 0.26 & 135 & 10 \\
$V$ & 0.58 & 0.11 & 168 & 5 & 0.79 & 0.16 & 137 & 6 \\
$R$ & 0.57 & 0.10 & 167 & 5 & 1.05 & 0.06 & 148 & 2 \\
$I$ & 0.45 & 0.14 & 188 & 9 & 0.55 & 0.14 & 147 & 7 \\
\hline
\end{tabular}

of polarisation for HR 5999 and HR 6864 is shown in Figs. 3 and 4 . The observed wavelength dependence does not fit the Serkowski formula. As can be seen from Figs. 3 and 4, the observed polarisation for HR 5999 decreases monotonically with wavelength from $B$ to $I$ band, while for HR 6864 it shows a sharp peak in the $R$ band and a steep fall from $R$ to the $I$ band. There is no polarisation peak in the $V$ band. This is unlike the interstellar polarisation. The observed variation of polarisation position angles with wavelength is only marginal for HR 5999 and HR 6864.

Another indication of the circumstellar nature of polarisation is its variability on short time scales. Only one of our programme stars (HR 3589) has been measured by us on more than one occasion. This A-type shell star showed $P_{V}=0.17 \pm 0.05 \%$ and $\theta_{V}=137 \pm 9^{\circ}$ on $27 \mathrm{Jan} 1993$ to be compared with $P_{V}=0.39 \pm 0.09 \%$ and $\theta_{V}=137 \pm 6^{\circ}$ on 1 January 1993 as listed in Table 1 . The percentage polarisation has undergone a very significant change on a time scale of a month, while the polarisation position angle stayed constant. The observed variation in polarisation can be understood in terms of variations in the distribution of scattering circumstellar material close to the star. We note that polarisation measurements have been made earlier, also for HR 4110 and HR 6631 (Serkowski et al. 1975) and HR 5999 (Bessell \& Eggen 1972; Hutchinson et al. 1994). The values of polarisation $P_{V}$ and position angle $\theta_{V}$ measured earlier are: $1.4 \%$ and $152^{\circ}$ for HR 4110 (compared with the values $2.06 \%$ and $146^{\circ}$ reported in the present work); $2.17 \%$ and $4.6^{\circ}$ for HR 6631 (present work: $1.92 \%$ and $5^{\circ}$ ); $0.46 \%$ and $6^{\circ}$ (Bessell \& Eggen 1972) and $1.19 \%$ and $13.4^{\circ}$ (Hutchinson et al. 1994) for HR 5999 (present work: $0.58 \%$ and $168^{\circ}$ ). Large variations in polarisation are shown by HR 4110 and HR 5999, while no significant variation is found for HR 6631. All the programme stars need further polarimetric monitoring and multi-wavelength measurements to study temporal variability and wavelength dependence of polarisation.

\section{Conclusions}

We have presented optical polarisation measurements for about 30 A-type stars from the Bright Star Catalogue that

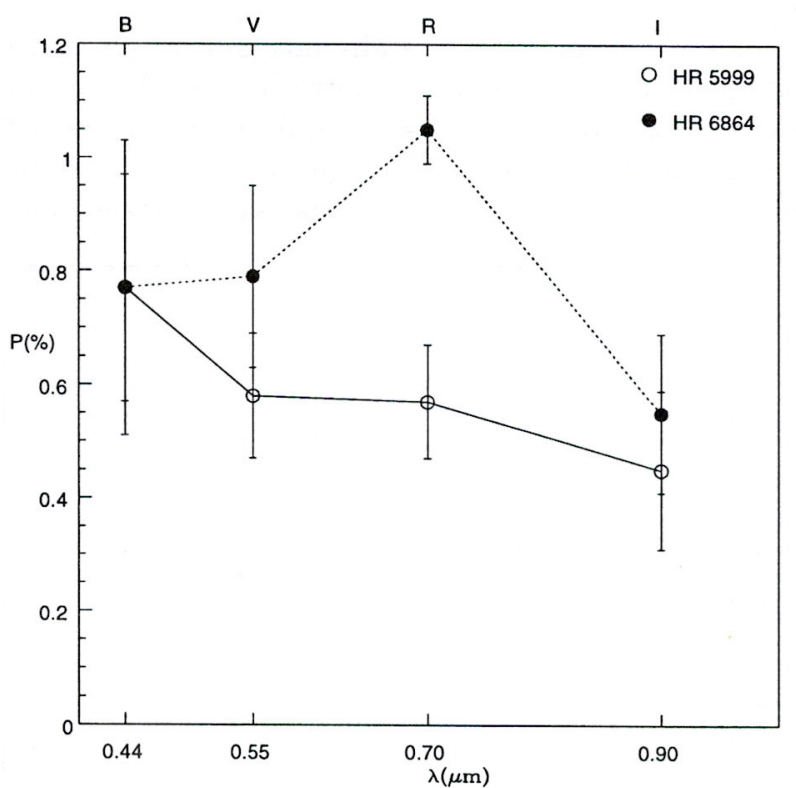

Fig. 3. Wavelength dependence of polarisation for HR 5999 and HR 6864

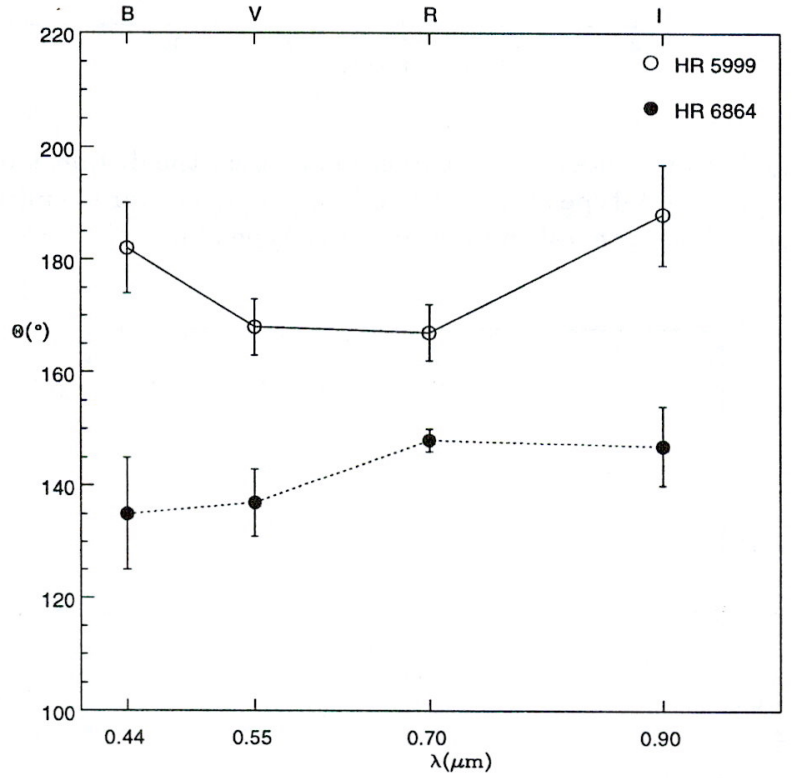

Fig. 4. Wavelength dependence of polarisation position angle for HR 5999 and HR 6864

are known to be either A-type shell stars or have shown anomalous emission in the infrared. The results show that these stars exhibit a degree of linear polarisation that is generally much larger than that shown by the normal Atype stars at similar distances, and can not be explained as interstellar polarisation. The observed polarisation also shows anomalous wavelength dependence and time variability for a few members of this sample of stars for which such measurements could be made. It is suggested that 
polarisation in the A-type shell stars and anomalous infrared emitters is circumstellar in origin. Further monitoring and multi-wavelength polarimetric observations of these stars would be very useful for a better understanding of the nature of their circumstellar shells.

\section{References}

Aumann H.H., 1985, PASP 97, 885

Bastien P., 1988, in Polarized Radiation of Circumstellar Origin. In: Coyne G.V., Moffat A.F.J., Tapia S., Magalhaes A.M., Schulte-Ladbeck R.E. and Wickramasinghe D.T. (eds.). Vatican Observatory Publication, p. 541

Bessell M.S., Eggen O.J., 1972, ApJ 177, 209

Coté J., 1987, A\&A 181, 77

Fernie J.D., 1974, PASP 86, 837

Hsu J.C., Breger M., 1982, ApJ 262, 732
Hutchinson M.G., Albinson J.S., Barrett P., et al., 1994, A\&A 285,883

Jain S.K., Srinivasulu G., 1991, Opt. Eng. 30, 1415

Jaschek M., Jaschek C., Egret D., 1986, A\&A 158, 325

Jaschek C., Jaschek M., Andrillat Y., Egret D., 1991, A\&A 252,229

Mathewson D.S., Ford V.L., 1970, Mem. RAS 74, 139

Schmidt-Kaler Th., 1965, Landolt-Bornstein, Vol. I of Astr. and Astrophys., Group VI. In: Voigt H.H. (ed.). SpringerVerlag, Berlin, p. 298

Serkowski K., 1973, in Interstellar Dust and Related Topics. In: Greenberg J.M. \& van de Hulst H.C. (eds.). Reidel, p. 145

Serkowski K., 1974, in Planets, Stars and Nebulae studied with photopolarimetry. In: Gehrels T. (ed.). University of Arizona Press, p. 135

Serkowski K., Mathewson D.S., Ford V.L., 1975, ApJ 196, 261 